\title{
Erratum: Inverse nodal problem for $p$-Laplacian energy-dependent Sturm-Liouville equation
}

Hikmet Koyunbakan*

\section{${ }^{*}$ Correspondence:}

hkoyunbakan@gmail.com Department of Mathematics, Firat University, Elazig, 23119, Turkey
In this note, we correct some mistakes in Theorem 2.1 and Theorem 2.2 which are given in Ref. [1].

Consider the problem (1.3), (1.4) in [1].

Theorem 2.1 [1] The eigenvalues $\lambda_{n}$ of the Dirichlet problem (1.3), (1.4) are

$$
\lambda_{n}^{2 / p}=n \pi_{p}+\frac{1}{p\left(n \pi_{p}\right)^{p-1}} \int_{0}^{1} q(t) d t+\frac{2}{p\left(n \pi_{p}\right)^{\frac{p-2}{2}}} \int_{0}^{1} r(t) d t+O\left(\frac{1}{n^{\frac{p}{2}}}\right) .
$$

Theorem 2.2 [1] For the problem (1.3), (1.4), the nodal point expansion satisfies

$$
\begin{aligned}
x_{j}^{n}= & \frac{j}{n}+\frac{j}{p n^{p+1}\left(\pi_{p}\right)^{p}} \int_{0}^{1} q(t) d t+\frac{2 j}{p n^{\frac{p}{2}+1}\left(\pi_{p}\right)^{\frac{p}{2}}} \int_{0}^{1} r(t) d t+\frac{2}{\left(n \pi_{p}\right)^{\frac{p}{2}}} \int_{0}^{x_{j}^{n}} r(x) S_{p}^{p} d x \\
& +\frac{1}{\left(n \pi_{p}\right)^{p}} \int_{0}^{x_{j}^{n}} q(x) S_{p}^{p} d x+O\left(\frac{1}{n^{\frac{p}{2}+2}}\right) .
\end{aligned}
$$

Proof Let $\lambda=\lambda_{n}$; integrating (2.3) from 0 to $x_{j}^{n}$, we have

$$
\frac{j \cdot \pi_{p}}{\lambda_{n}^{2 / p}}=x_{j}^{n}-\int_{0}^{x_{j}^{n}} \frac{2 r(x)}{\lambda_{n}} S_{p}^{p} d x-\int_{0}^{x_{j}^{n}} \frac{q(x)}{\lambda_{n}^{2}} S_{p}^{p} d x .
$$

By using the estimates of eigenvalues as

$$
\frac{1}{\lambda_{n}^{2 / p}}=\frac{1}{n \pi_{p}}+\frac{1}{p\left(n \pi_{p}\right)^{p+1}} \int_{0}^{1} q(t) d t+\frac{2}{p\left(n \pi_{p}\right)^{\frac{p}{2}+1}} \int_{0}^{1} r(t) d t+O\left(\frac{1}{n^{\frac{p}{2}+2}}\right),
$$

we obtain the result.

Received: 19 September 2014 Accepted: 19 September 2014 Published online: 01 October 2014

\section{Reference}

1. Koyunbakan, H: Inverse nodal problem for $p$-Laplacian energy-dependent Sturm-Liouville equation. Bound. Value Probl. 2013, 272 (2013). doi:10.1186/1687-2770-2013-272

( 2014 Koyunbakan; licensee Springer. This is an Open Access article distributed under the terms of the Creative Commons Attribution License (http://creativecommons.org/licenses/by/4.0), which permits unrestricted use, distribution, and reproduction in any medium, provided the original work is properly credited. 
doi:10.1186/s13661-014-0222-3

Cite this article as: Koyunbakan: Erratum: Inverse nodal problem for $p$-Laplacian energy-dependent Sturm-Liouville equation. Boundary Value Problems 2014 2014:222.

Submit your manuscript to a SpringerOpen ${ }^{\circ}$ journal and benefit from:

- Convenient online submission

- Rigorous peer review

- Immediate publication on acceptance

- Open access: articles freely available online

- High visibility within the field

- Retaining the copyright to your article

Submit your next manuscript at $\gg$ springeropen.com 